\title{
Tracing the role of sucrose in potato microtuber formation in vitro
}

\author{
M.S. Islam ${ }^{1}$, M.Z.K. Roni ${ }^{2}$, A.F.M. Jamal Uddin ${ }^{3}$ and Kazuhiko Shimasaki ${ }^{4}$ \\ ${ }^{1}$ The United Graduate School of Agricultural Sciences, Ehime University, 3-5-7 Tarumi, Matsuyama, Ehime 790- \\ 8566, Japan \\ ${ }^{2}$ The United Graduate School of Agricultural Sciences, Ehime University, 3-5-7 Tarumi, Matsuyama, Ehime 790- \\ 8566, Japan \\ ${ }^{3}$ Department of Horticulture, Sher-e-Bangla Agricultural University, Dhaka-1207, Bangladesh \\ ${ }^{4}$ Faculty of Agriculture, Kochi University, B 200 Monobe, Nankoku, Kochi 783-8502, Japan
}

*Corresponding author: saiful1236@gmail.com

\begin{abstract}
Sucrose is a necessary external carbon substrate for in vitro microtuber induction and development. In this study, we determined the correlation between sucrose and microtuber formation in potato by investigating the role and significance of sucrose in the medium and the origin of explants, as well as the potential function of sucrose in microtuber formation in vitro. Sucrose strongly influences microtuber induction, growth, and earliness without negative side effects. The results of this study show there was a significant correlation of $R^{2}=0.95$ between tuberization and high sucrose content in the medium. High sucrose content in the medium is the carbon source that influences microtuber formation and development irrespective of the origin of explants. The present work could be considered efficient for large scale multiplication and propagation of this important vegetable crop in vitro. Hence, the results of this study should help rapid micropropagation of commercial potato cultivars by using high concentrations of sucrose in the microtuberization medium for a higher percentage of microtuber formation with earliness.
\end{abstract}

Key words: Correlation, In vitro, Microtuber, Sucrose concentration, Solanum tuberosum.

\section{Introduction}

Potato (Solanum tuberosum L.) is one of the world's most important crops (FAO, 1995) and is one of the main vegetable crops in Bangladesh (Hoque, 2010). Therefore, the potential value of tissue culture technology in potato microtuber production has been broadly recognized (Wattimena, 1995). Tuberization in potato is a highly complex developmental process that can be induced under in vitro conditions throughout the year (Hoque, 2010). Furthermore, microtubers are very easy to carry and can be stored for a long time because they are small and cut weight (Nistor et al., 2010; Sakha et al., 2004; Coleman et al., 2001). In addition, they can be planted directly in the soil as a seed and can be produced during any period of the year with similar morphology and biochemical features to traditional tubers (Nistor et al., 2010).

There have been numerous studies on potato microtuber production, but they have little bearing on the in vitro tuberization process from different origins of explants, and most of the studies have used complex media containing plant growth regulators that affect microtuber induction and dormancy (Coleman et al., 2001). However, it is clear that the most important factors during tuber formation period are a) sugar (sucrose) concentration in the culture medium; b) nitrogen content (interaction between sugar and nitrogen); and c) temperature and light (Nistor et al., 2010; El-Sawy et al., 2007; Ranalli, 2007). Of the three factors, sucrose concentration is the most important stimulus for tuber induction and development of potato tubers (Nistor et al., 2010; El-Sawy et al., 2007). High sucrose concentration is essential for in vitro microtuber induction (Nistor et al., 2010; Khuri and Moorby, 1995). However, little is known about the correlation between sucrose and tuberization resulting from different origins of explants and various external and internal factors.

Sucrose has been shown to be a necessary external carbon substrate for in vitro microtuber induction and development (Nistor et al., 2010; El-Sawy et al., 2007; Coleman et al., 2001; Gopal et al., 2004; Khuri and Moorby, 1995). In this study, we determined the correlation between sucrose and microtuber formation in potato by investigating the role and significance of sucrose in the medium and the origin of explants, as well as the potential function of sucrose in microtuber formation in vitro.

\section{Results}

\section{Influence of sucrose concentration on tuberization in vitro}

During the culture period, various factors influenced the induction and growth of microtubers. The influence of different sucrose concentrations $(1,3,5$, and $8 \%$ ) on microtuber formation was studied to determine the correlation between the two. Higher tuberization percentages were observed with higher percentages of sucrose concentration 
BARI TPS-1

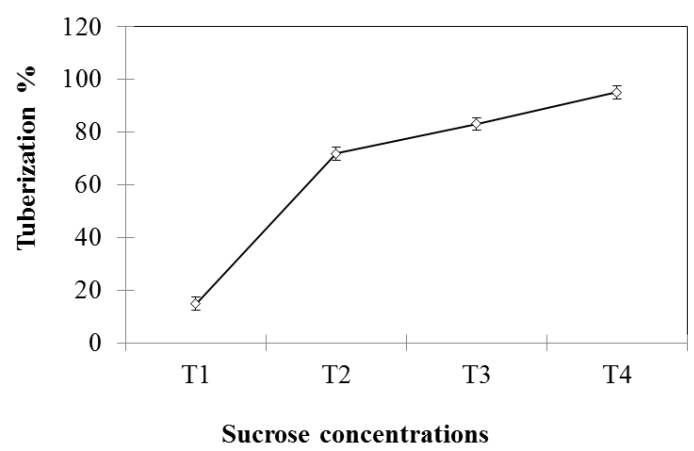

cv. Diamant

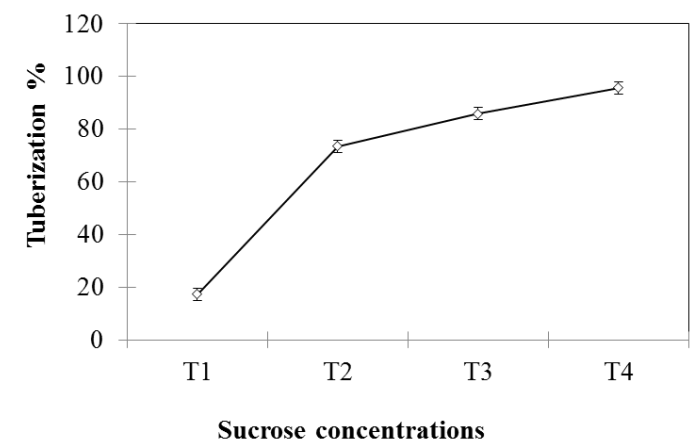

Fig 1. Influence of sucrose concentration on tuberization percentage (Tuberization percentages were calculated as percentages of microtubers formed in different cultures: $\mathrm{T}_{1}-1 \%$ sucrose, $\mathrm{T}_{2}-3 \%$ sucrose, $\mathrm{T}_{3}-5 \%$ sucrose, and $\mathrm{T}_{4}-8 \%$ sucrose).

BARI TPS - 1

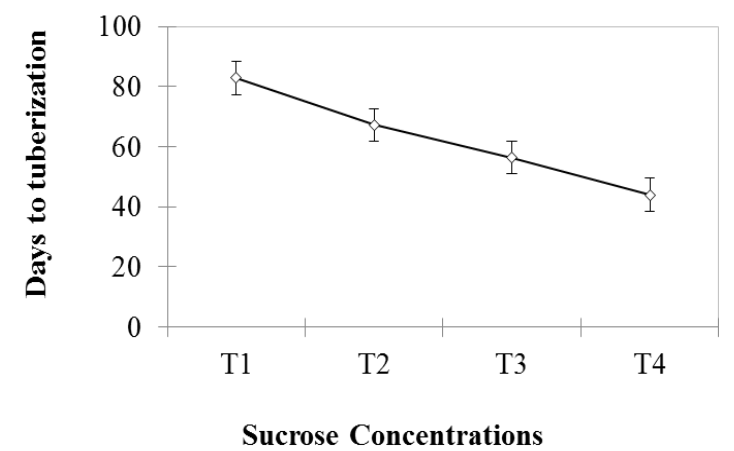

cv. Diamant

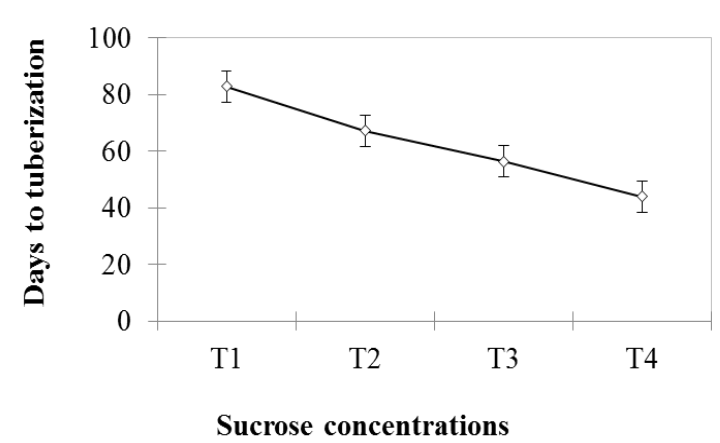

Fig 2. Influence of sucrose concentration on time to microtuber formation (Tuberization percentages were calculated as percentages of microtubers formed in different cultures: $\mathrm{T}_{1}-1 \%$ sucrose, $\mathrm{T}_{2}-3 \%$ sucrose, $\mathrm{T}_{3}-5 \%$ sucrose, and $\mathrm{T}_{4}-8 \%$ sucrose).

BARI TPS-1

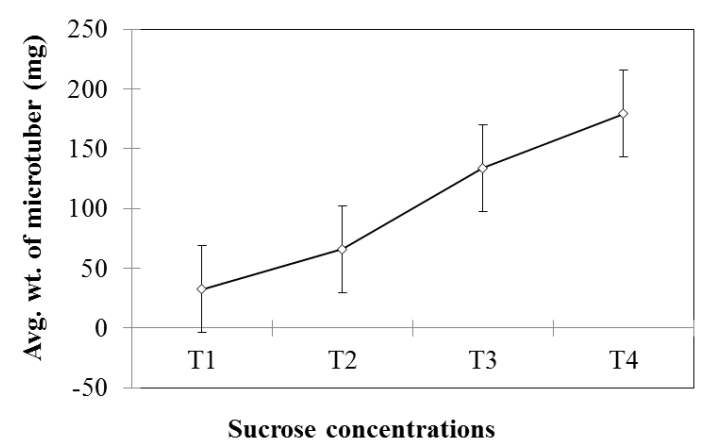

cv. Diamant

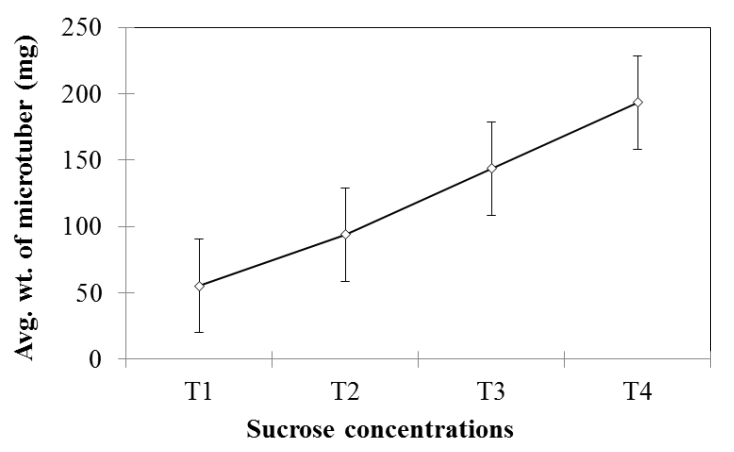

Fig 3. Influence of sucrose concentration on fresh weight of microtubers $\left(T_{1}-1 \%\right.$ sucrose, $T_{2}-3 \%$ sucrose, $T_{3}-5 \%$ sucrose, and $T_{4}$ $8 \%$ sucrose). 
BARI TPS-1

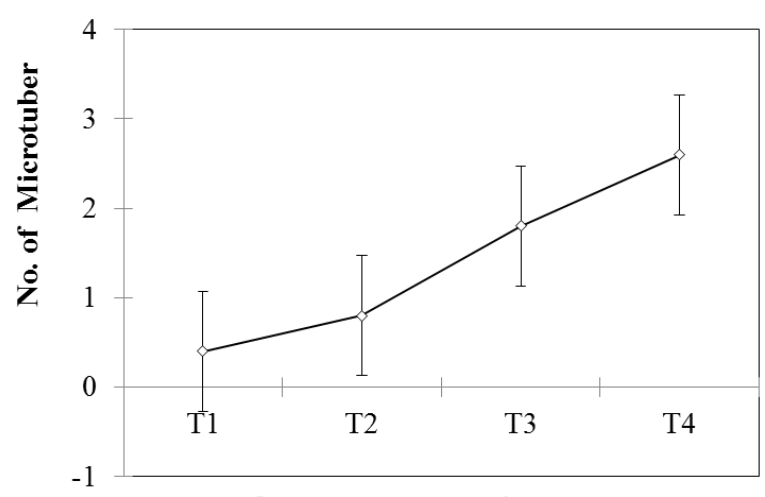

Sucrose concentrations

\section{cv. Diamant}

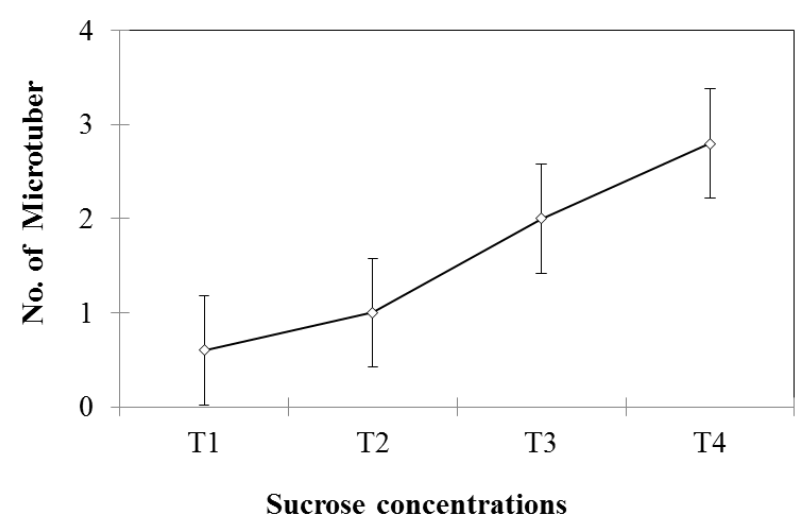

Fig 4. Influence of sucrose concentration on microtubers per explant $\left(\mathrm{T}_{1}-1 \%\right.$ sucrose, $\mathrm{T}_{2}-3 \%$ sucrose, $\mathrm{T}_{3}-5 \%$ sucrose, and $\mathrm{T}_{4}-8 \%$ sucrose).

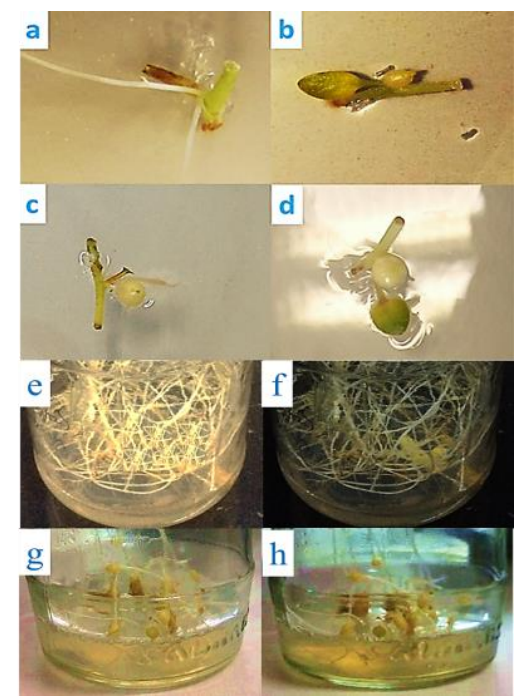

Fig 5. Development of microtubers in relation to sucrose concentration in medium. a) no microtuber from BARI TPS-1 with $1 \%$ sucrose, b) microtuber from BARI TPS-1 with 3\% sucrose, c) microtuber from BARI TPS-1 with 5\% sucrose, d) microtuber from BARI TPS-1 with $8 \%$ sucrose, e) no microtuber from cv. Diamant with $1 \%$ sucrose, f) microtuber from cv. Diamant with $3 \%$ sucrose, g) microtuber from cv. Diamant with 5\% sucrose h) microtuber from cv. Diamant with $8 \%$ sucrose.

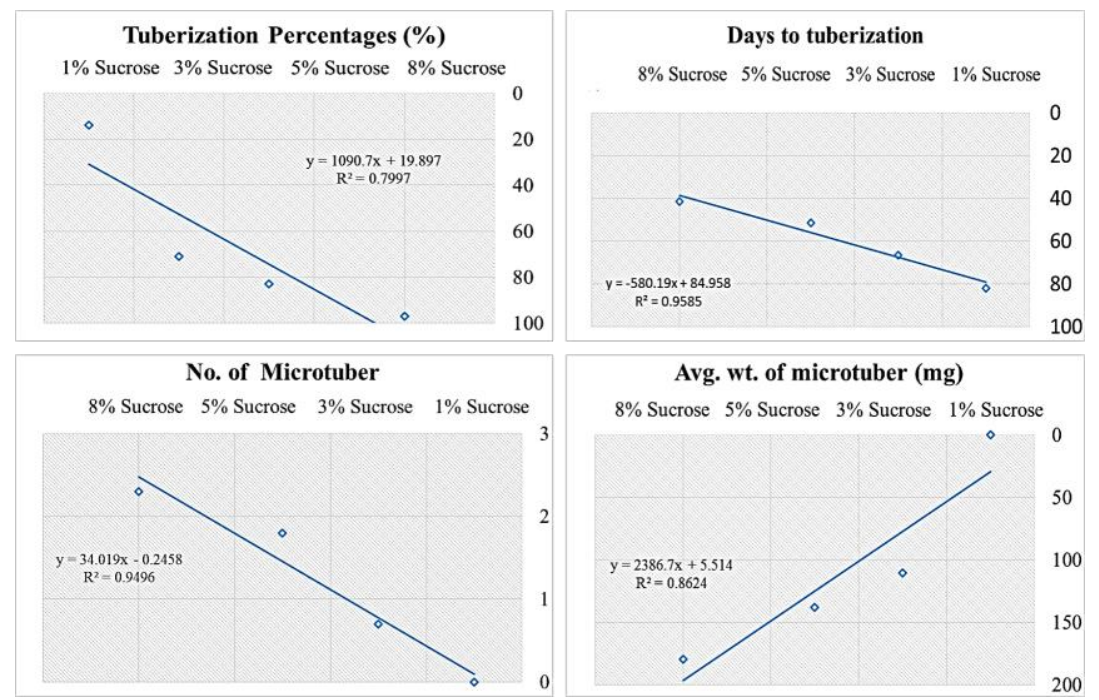

Fig 6. Correlation between tuberization and sucrose concentration in the medium (Mean data for all treatments used to diagnose the correlation) 
(Fig. 1), irrespective of the origin of explants (either BARI TPS-1 or cv. Diamant). Accordingly, higher sucrose concentrations enhanced the tuberization percentages in vitro.

\section{Influence of sucrose concentration on time to tuberization}

The influence of different sucrose concentrations (1, 3, 5, and $8 \%$ ) was investigated by comparing the days to microtuber formation in vitro. Figure 2 shows that both explants exhibit similar patterns of time to tuberization. For BARI TPS-1, $8 \%$ resulted in minimum days to tuberization, with $1 \%$ for maximum days. For cv. Diamant, $8 \%$ resulted in minimum days to tuberization, with $1 \%$ for maximum days. Hence, sucrose concentration significantly affects time to tuberization in vitro and its effect may be greatest mainly during tuber induction and development.

\section{Influence of sucrose concentration on fresh weight of microtuber}

The influence of sucrose concentration $(1,3,5$, and $8 \%)$ was studied to determine the correlation between fresh weight of microtubers and sucrose. As the results show, there is a linear relationship between fresh weight of microtubers and different sucrose concentrations. The higher the sucrose concentration, the bigger the microtuber and thus higher fresh weight (Fig. 3), irrespective of the origin of explants (either BARI TPS- 1 or cv. Diamant).

\section{Influence of sucrose concentration on microtubers per explant}

The correlation between sucrose concentration $(1,3,5$, and $8 \%$ ) and microtubers per explant was studied by comparing the number of microtubers per explant in vitro. The results show a linear relationship between the number of microtubers per explant and different sucrose concentrations. The higher the sucrose concentration, the higher the number of microtubers per explant (Fig. 4), irrespective of the origin of explants (either BARI TPS-1 or cv. Diamant).

\section{Discussion}

Higher sucrose concentration in the medium of an in vitro culture is one of the most important factors in inducing microtubers (Podwyszyěska, 2012; Xu et al., 1998 a, b). The growth of microtubers in relation to different sucrose concentrations $(1,3,5$, and $8 \%)$ in this study raised the question of how sucrose triggered the stimulus for tuber induction and development in vitro, because there was a clear visual difference between microtubers with different sucrose concentrations (Fig. 5). Moreover, there was a significant correlation $\left(\mathrm{R}^{2}=0.95\right)$ between tuberization and sucrose content in the medium (Fig. 6). Sucrose, in high concentrations in vitro, act both internal and external factors (Podwyszyěska, 2012) is the most important organic nutrient affecting the rate and percentage of tuberization (El-Sawy et al., 2007; Wattimena, 1995). An increasing concentration of sucrose results in an increased percentage and earliness of tuberization (El-Sawy et al., 2007; Wattimena, 1995) of induced tissue. According to Momena et al. (2014) and Sarkar et al. (2006), BAP has a crucial role in determining the size of microtubers, because the growth of microtubers is stimulated by a high sucrose concentration with low nitrogen. High levels of carbohydrates in the form of sugars and starch favor the formation of storage organs such as tubers, whereas high nitrogen levels are known to promote shoot growth
(Aksenova et al., 2012; Podwyszyěska, 2012; Koda and Okazawa, 1983). In investigating these relationships, we recognized the contribution of higher sucrose on tuberization in the medium (Gopal et al., 2004; Coleman et al., 2001). Hence, sucrose concentration and tuberization exhibit a strong correlation. Likewise, El-Sawy et al. (2007), Coleman et al. (2001), and Wattimena (1995) in their studies also found that increased sucrose concentration increased tuberization with earliness. However, a high sucrose concentration also correlated with microtuber size because of higher expression of the cell cycle (Podwyszyěska, 2012; Koda and Okazawa, 1983), which suggested that sucrose may in this way influence cell division at stolon swellings (Aksenova et al., 2012; Xu et al., 1998 a, b). During the transition to tuber induction and the swelling stage, there is a switch in sucrose metabolism in the stolon sub-apical region (Alisdair and Willmitzer, 2001), triggering a sequence of biochemical, physiological, and morphological events leading to storage organ formation. Growing potato tubers are storage sinks in the tubers where carbohydrates are deposited in the form of starch and mainly accumulates in the cortex, phloem parenchyma, and external pith layers in potato tubers (Geigenberger, 2003).

\section{Materials and Methods}

The experiment was conducted at the 2a biotech lab, Sher-eBangla Agricultural University, Dhaka-1207, Bangladesh from March to September, 2015.

\section{Preparation of explants}

Two different origins of plant material were used. 1) In vitro potato plantlets from BARI TPS-1. The TPS were collected from the Tuber Research Centre, Bangladesh Agriculture Research Institute, Gazipur. The variety is a hybrid between a female parent (MF-II) and a male parent (TPS-67) that was released by the National Seed Board (NSB) in 1997. Those TPS were germinated, grown, and cultured on MS medium (Nizamuddin et al., 2010) in a firm and clear plastic tissue culture container $(18 \times 12 \times 12 \mathrm{~cm})$ made of highly purified polypropylene, which provides excellent light transmission. The vent at the top of the cover was sealed by a PTFE filter membrane (pore size: $0.45 \mu \mathrm{m}$ ). The container was incubated at $25 \pm 2^{\circ} \mathrm{C}$ under $16 \mathrm{~h}$ of light at an intensity of $2.5 \times 10^{2} \mathrm{~lx}$. The source of light was fluorescent tubes. Within four weeks, the plantlets were ready for further culture to tuber induction. Explants from those plantlets were used in this experiment. 2) In vitro potato plantlets (cv. Diamant). Potato tubers were washed 3 times with detergent (Tween 20-polysorbate surfactant) followed by rinsing with distilled water. After washing, tubers were soaked in GA3 $50 \mathrm{mg} / \mathrm{L}$ for $6 \mathrm{~h}$. The GA3-treated tubers were then dried and kept in a dark room at around $25{ }^{\circ} \mathrm{C}$ for a week for rapid sprouting. Healthy sprouts were taken and washed with detergent (Tween 20polysorbate surfactant) followed by rinsing with distilled water. After washing with detergent, sprouts were again washed with $70 \%$ ethanol for 30 s inside a laminar airflow cabinet for further sterilization to avoid pathogenic contamination. The sprouts were then rinsed twice with autoclaved double-distilled water for $30 \mathrm{~min}$.

\section{In vitro culture condition}

After sterilization, the sprouts were placed on the MS basal medium supplemented with $2 \mathrm{mg} / \mathrm{L}$ BAP (benzyladenin) and $0.5 \mathrm{mg} / \mathrm{L}$ GA3 for shoot initiation. After 4 weeks, buds 
sprouted into plantlets having 5-7 nodes. Single node cuttings $(1.5 \mathrm{~cm}$, without leaves and apical meristem) were excised and used as explants (Momena et al., 2014). Three BARI TPS-1 explants were placed in a plastic Petri dish and three cv. Diamant explants in a glass vessel, both containing Murashige and Skoog (MS) basal medium with $10 \%$ of the standard amount of nitrogen (tuber-inducing MS: $1.20 \mathrm{~g} / \mathrm{L}$ ) supplemented with $5 \mathrm{mg} / \mathrm{L}$ BAP (benzyladenin) with sucrose $\left(1 \%, 10 \mathrm{~g} / \mathrm{L}\left\{\mathrm{T}_{1}\right\} ; 3 \%, 30 \mathrm{~g} / \mathrm{L}\left\{\mathrm{T}_{2}\right\} ; 5 \%, 50 \mathrm{~g} / \mathrm{L}\left\{\mathrm{T}_{3}\right\}\right.$; and $\left.8 \%, 80 \mathrm{~g} / \mathrm{L}\left\{\mathrm{T}_{4}\right\}\right)$ at $\mathrm{pH} 5.8$ and agar $7 \mathrm{~g} / \mathrm{L}$ for in vitro tuberization.

\section{Statistics}

For each sucrose concentration, 30 replications were taken and placed in the dark chamber at $20^{\circ} \mathrm{C}$ for microtuber induction. The results are expressed as mean \pm standard error (SE). For all comparisons, statistical analysis was performed using one-way ANOVA, and $\mathrm{p}<0.05$ was considered statistically significant.

\section{Conclusion}

Microtubers grown in an aseptic manner on defined nutrient media emerged as the propagules of choice in various studies on potatoes. High concentrations of sucrose in the medium added a carbon source, which influences microtuber formation and development, irrespective of the origins of explants. Hence, sucrose has a positive influence on tuberization in vitro, and therefore, higher concentrations of sucrose contributed to the highest percentage of microtuber formation with earliness.

\section{Acknowledgement}

We thank Dr. AFM Jamal Uddin and Dr. Kazuhiko Shimasaki for their support, stimulating discussions, and helpful comments. We are also grateful for the support of the 2a biotech lab, Sher-e-Bangla Agricultural University, Dhaka-1207, Bangladesh.

\section{References}

Aksenova NP, Konstantinova TN, Golyanovskaya SA, Sergeeva LI, Romanov GA (2012) Hormonal regulation of tuber formation in potato plants. Russ J Plant Physiol. 59: 451-466.

Alisdair RF, Willmitzer L (2001) Molecular and biochemical triggers of potato. Plant Physiol. 127: 1459-1465.

Coleman WK, Donnelly DJ, Coleman SE (2001) Potato microtubers as research tools: A review. Am J Potato Res. 78(1): 47-55

El-Sawy A, Bekheet S, Aly UI (2007) Morphological and molecular charecterization of potato microtubers production on coumarin inducing medium. In J Agri Biol. 9(5): 675-680.
FAO (1995) Statistical summary of agricultural production. In: Quarterly bulletin of statistics. Food and Agric Org of the United Nations, Rome. 5(1): 14-17.

Geigenberger P (2003) Regulation of sucrose to starch conversion in growing potato tubers. J Exp Bot. 54(382): 457-465.

Gopal J, Chamail A, Sarkar D (2004) In vitro production of microtubers for conservation of potato germplasm: Effect of genotype, abscisic acid, and sucrose. In Vitro Cell Dev Biol Plant. 40(5): 485-490.

Hoque ME (2010) In vitro tuberization in potato (Solanum tuberosum L .). Plant Omics Journal. 3(1): 7-11.

Khuri S, Moorby J (1995) Investigations into the role of sucrose in potato cv. Estima microtuber production in vitro. Annals of Botany. 75: 295-303.

Koda Y, Okazawa Y (1983) Influences of environmental, hormonal and nutritional factors on potato tuberization in vitro. Jpn J Crop Sci. 52: 582-591.

Momena K, Adeeba R, Mehraz H, Jamal Uddin AFM, Islam S, Rahman L (2014) In vitro microtuberization of potato (Solanum tuberosum L.) cultivar through sucrose and growth regulator. J Biosci Agric Res. 2(2): 76-82.

Nistor A, Campeanu G, Atanasiu N, Karacsonyi (2010) Influence of potato genotypes on "in vitro" production of microtubers. Rom Biotech Lett. 15(3): 5317-5324.

Nizamuddin, Maqsood Q, Sher A, Bushra M, Muhammad D, Iqbal H (2010) In vitro response of true potato seed (TPS) parents for micro propagation. Sarhad J Agri. 26(2): 245249.

Podwyszyěska M (2012) The mechanisms of in vitro storage organ formation in ornamental geophytes. Floriculture and Ornamental Biotechnology. 6(1): 9-23.

Ranalli P (2007) The canon of potato science:24. Microtubers Potato Research. 50: 301-304.

Sakha BM, Bhatia AK, Batra VK, Chaudhary VK, Batra P, Khurana SC (2004) In vitro microtuberization in potato (Solanum tuberosum L.) cultivars. Indian J Exp Biol. 42(12): 1245-1247.

Sarkar D, Pandey SK, Sharma S (2006) Cytokinins antagonize the jasmonates action on the regulation of potato (Solanum tuberosum) tuber formation in vitro. Plant Cell Tiss Org. 87: 285-295.

Wattimena G A (1995) In vitro microtubers as an alternative technology for potato production. Department of Agronomy, Faculty of Agriculture, Bogor Agricultural University (IP), Bogor Indonesia and Department of Horticulture University of Wisconsin, Madison, USA, 1995.

Xu X, Van Kammeren AAM, Vermeer E, Vreugdenhil D (1998a) The role of gibberellin, absicic acid, and sucrose in the regulation of potato tuber formation in vitro. Plant Physiol. 117: 575-584.

Xu X, Vreugdenhil D, Van Lammeren AAM (1998b) Cell division and cell enlargement during potato tuber formation. J Exp Bot. 49: 573-582. 